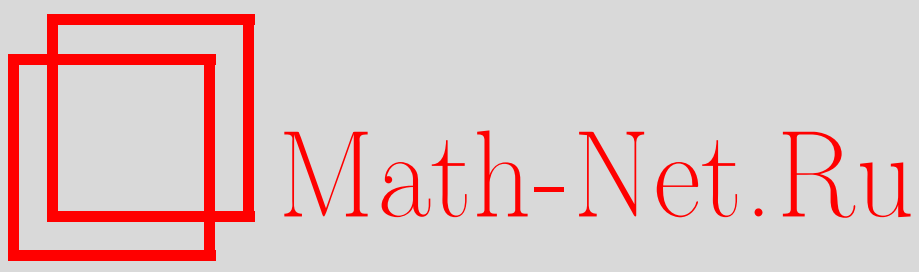

В. П. Бурский, Обобщенные решения линейных граничных задач, Изв. вузов. Матем., 2019, номер 12, 25-36

DOI: https://doi.org/10.26907/0021-3446-2019-12-25-36

Использование Общероссийского математического портала Math-Net.Ru подразумевает, что вы прочитали и согласны с пользовательским соглашением

http: //www.mathnet.ru/rus/agreement

Параметры загрузки:

IP : 3.93 .64 .190

26 апреля 2023 г., $12: 11: 02$

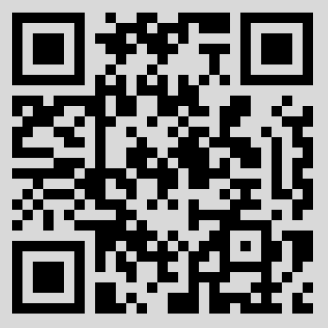


Известия вузов. Математика

2019, № 12 , c. 25-36 https://kpfu.ru/science/nauchnye-izdaniya/ivrm

e-mail: izvuz.matem@kpfu.ru

\title{
В.П. БУРСКИЙ
}

\section{ОБОБЩЕННЫЕ РЕШЕНИЯ ЛИНЕЙНЫХ ГРАНИЧНЫХ ЗАДАЧ}

\begin{abstract}
Аннотация. Используются конструкции из общей теории граничных задач для построения теории обобщенных граничных задач для обобщенного уравнения Пуассона. А именно, вводятся и изучаются обобщенные решения различных граничных задач для матричного обобщения уравнения Пуассона, приведено описание множества всех таких корректных задач. Полученные результаты используются для продвижений в исходной общей теории граничных задач.
\end{abstract}

Ключевые слова: общая теория граничных задач, расширения дифференциальных операторов, обобщенные постановки, обобщенные решения.

УДК: 517.95

DOI: $10.26907 / 0021-3446-2019-12-25-36$

\section{ВВЕДЕНИЕ}

Основы общей теории граничных задач безотносительно к типу уравнения были заложены в известной работе М.Й. Вишика [1], в которой граничная задача проявляется в задании области определения некоторого расширения минимального оператора. Следующим важным шагом в развитии теории явилось уточнение понятия граничной задачи вместе с интерпретацией результатов работы [1] и доказательством условий М.Й. Вишика для скалярных дифференциальных операторов с постоянными коэффициентами, данные Л. Хёрмандером в ([2], с. 13-29). После бума всеобщего интереса 60-х годов к этой тематике и осознания трудностей, связанных с отсутствием серьезных продвижений в исследованиях, в интересе аналитиков к этой области наблюдался длительный спад. Отметим среди работ 60-х годов исследования Ю.М. Березанского ([3], с. 93-115) по гладкопорожденным общим граничным задачам, М.С.Аграновича [4] и позже А.А.Дезина [5]. В настоящее время изучение общих постановок граничных задач проходит в направлениях, заданных еще в середине 20-го столетия И.Г. Петровским [6] и Я.Б. Лопатинским [7], когда постановки граничных задач увязывались с конкретным типом дифференциального уравнения, и среди таких постановок актуальным вопросом является выделение корректных постановок граничных задач (например, книга А.В. Бицадзе [8], монография А.П. Солдатова, начало в [9]). Возникли также разные обобщенные постановки граничных задач, в частности, теория обобщенных решений граничных задач, опирающаяся на теорию оснащенных пространств, сейчас такие задачи ставятся для линейных и квазилинейных дивергентных уравнений эллиптического типа, а также и для других типов уравнений, в которых эллиптическая часть выражена дивергентными членами, т. е. развитие происходит в направлении, заданном еще в работах С.Л. Соболева и О.А. Ладыженской (например, [10], с. 91-114). В настоящей работе методы, наработанные в общей теории граничных задач, применяются к уравнению $\mathcal{L}^{+} \mathcal{L}=f$,

Поступила в редакцию 25.12.2018, после доработки 25.12.2018. Принята к публикации 27.03.2019. 
где $\mathcal{L}$ - общий матричный линейный дифференциальный оператор, a $\mathcal{L}^{+}-$его формально сопряженный. При этом идея постановки обобщенной граничной задачи для уравнения дивергентного вида преобразуется в идею похожей постановки с уравнением $\mathcal{L}^{+} \mathcal{L}=f$, что развивает теорию обобщенных решений в направлении теории расширений. Полезность этих обобщений демонстрируется на примере приложений к общей теории граничных задач. Настоящая работа продолжает исследования, начатые автором в статьях [11]-[13].

\section{1. СВЕДЕНИЯ ИЗ ОБЩЕЙ ТЕОРИИ ГРАНИЧНЫХ ЗАДАЧ}

Пусть $\mathcal{L}=\sum_{|\alpha| \leq l} a_{\alpha}(x) D^{\alpha}, \quad a_{\alpha} \in C^{\infty}(\bar{\Omega}), \quad D^{\alpha}=\frac{(-i \partial)^{|\alpha|}}{\partial x^{\alpha}}-$ дифференциальная операция общего вида с $j \times k$-матрицами $a_{\alpha}$, элементами которых являются гладкие комплекснозначные функции, и пусть $\Omega$ - произвольная ограниченная область в $\mathbb{R}^{n}$. Операция $\mathcal{L}$ порождает формально сопряженную операцию $\mathcal{L}^{+}=\sum_{|\alpha| \leq l} D^{\alpha}\left(a_{\alpha}^{*}(x) \cdot\right)$, где $a_{\alpha}^{*}(x)-$ сопряженная матрица. Будем обозначать $H=L_{2}^{j}(\Omega), H^{+}=L_{2}^{k}(\Omega), H^{l}=\left(H^{l}(\Omega)\right)^{j}, H_{0}^{l}=\left(H_{0}^{l}(\Omega)\right)^{j}$, $H^{+l}=\left(H^{l}(\Omega)\right)^{k}, H_{0}^{+l}=\left(H_{0}^{l}(\Omega)\right)^{k}-$ соболевские пространства.

В этой статье будут рассматриваться обобщенные постановки граничных задач для уравнений вида

$$
\begin{aligned}
\mathcal{L}^{+} \mathcal{L} u & =f, \\
\mathcal{L} \mathcal{L}^{+} u & =f .
\end{aligned}
$$

Примером такого уравнения может служить уравнение Пуассона $-\operatorname{div} \operatorname{grad} u=f$.

Минимальный оператор $L_{0}$, определяемый как замыкание оператора $\mathcal{L}$, первоначально заданного на $\left(C_{0}^{\infty}(\Omega)\right)^{j}$, в норме графика $\|u\|_{L}^{2}=\|u\|_{L_{2}^{j}(\Omega)}^{2}+\|\mathcal{L} u\|_{L_{2}^{k}(\Omega)}^{2}$, и аналогичный минимальный оператор $L_{0}^{+}$порождают максимальные операторы $L=\left(L_{0}^{+}\right)^{*}, L^{+}=L_{0}^{*}$ с помощью сопряжения в гильбертовых пространствах. Области определения $D\left(L_{0}\right), D\left(L_{0}^{+}\right)$, $D(L), D\left(L^{+}\right)$этих операторов являются гильбертовыми пространствами в соответствующей норме графика.

Введем граничное пространство $C(L)=D(L) / D\left(L_{0}\right)$ для оператора $L$, а также факторотображение $\Gamma: D(L) \rightarrow C(L)$ и аналогично $C\left(L^{+}\right)$и $\Gamma^{+}-$для оператора $L^{+}$. Для максимального оператора $L$ имеем короткую точную последовательность ([14], с. 22)

$$
0 \rightarrow \operatorname{ker} L \rightarrow D(L) \rightarrow \operatorname{Im} L \rightarrow 0 .
$$

Имеется похожая последовательность для минимального оператора, а кроме того, точные последовательности факторизации $\operatorname{Im} L / \operatorname{Im} L_{0}$ и $C(L)=D(L) / D\left(L_{0}\right)$. Собрав это вместе, получим диаграмму

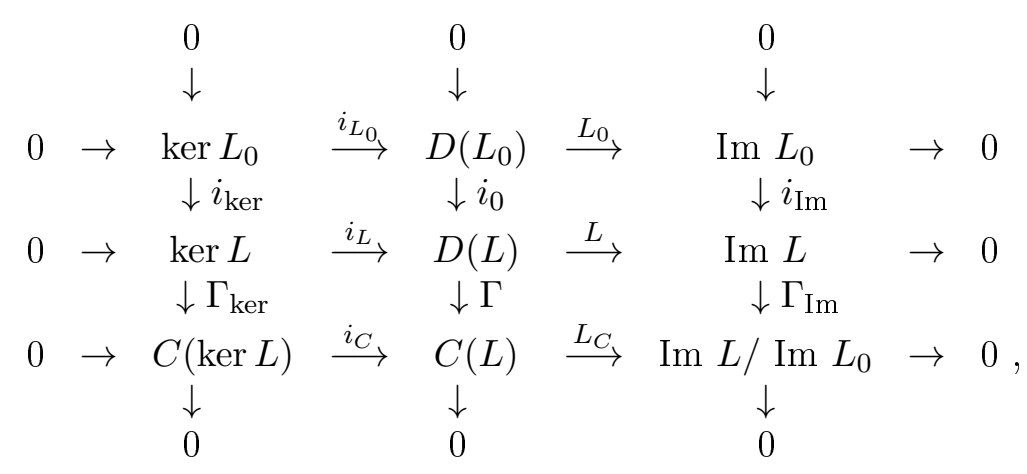


где операторы $i_{C}$ и $L_{C}$ определены формулами $i_{C}\left(u+\operatorname{ker} L_{0}\right)=u+D\left(L_{0}\right), L_{C}\left(u+D\left(L_{0}\right)\right)=$ $L u+\operatorname{Im}\left(L_{0}\right)$, а оператор $\Gamma_{\text {ker }}: \operatorname{ker} L \rightarrow C(\operatorname{ker} L):=\operatorname{ker} L / \operatorname{ker} L_{0}$ - отображение факторизации.

Коммутативность всех квадратов очевидна. Таким образом, диаграмма (3) коммутативна, все столбцы и две верхние строки точны. Из алгебраической $3 \times 3$-леммы ([14], с. 72) получаем точность нижней строки. Доказана

Теорема 1. Диаграмма (3) коммутативна, ее строки и столбиы точны.

Диаграмма (3), очевидно, означает разложение в прямую сумму $L=L_{0} \oplus L_{C}$.

Рассмотрим следующие условия Вишика [1]:

$$
\begin{aligned}
& \text { оператор } L_{0}: D\left(L_{0}\right) \rightarrow H^{+} \text {имеет непрерывный левый обратный, } \\
& \text { оператор } L_{0}^{+}: D\left(L_{0}^{+}\right) \rightarrow H \text { имеет непрерывный левый обратный. }
\end{aligned}
$$

Как следует из стандартных рассуждений функционального анализа эти утверждения эквивалентны соответственно утверждениям $(4) \approx\left(4^{\prime}\right),(5) \approx\left(5^{\prime}\right)$ :

$$
\begin{aligned}
& \text { оператор } L: D(L) \rightarrow H^{+} \text {сюрьективен, } \\
& \text { оператор } L^{+}: D\left(L^{+}\right) \rightarrow H \text { сюрьективен. }
\end{aligned}
$$

Пример 1. В статье [11] были указаны некоторые классы дифференциальных операторов, для которых выполнены условия (4), (5) в ограниченной области. В этом списке были

i) скалярные операторы с постоянными коэффициентами,

ii) скалярные операторы главного типа,

iii) скалярные операторы постоянной силы,

iv) матричные операторы с постоянными комплексными коэффициентами со свойством Панеяха-Фугледе,

v) матричные операторы, равномерно эллиптические по Дуглису-Ниренбергу в области с гладкой границей.

Из условия (4) следует $\operatorname{ker} L_{0}=0$, а условие (5) влечет $\operatorname{Im} L=H$. Кроме того, известно, что для замкнутого $\operatorname{Im} L_{0}$ имеет место разложение $H^{+}=\operatorname{Im} L_{0} \oplus \operatorname{ker} L^{+}$. Таким образом, в условиях (4), (5) диаграмма (3) превращается в диаграмму

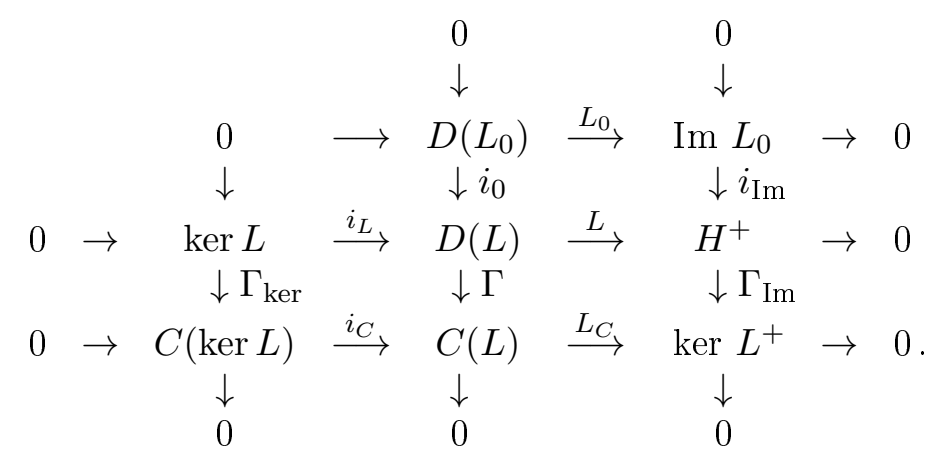

Однородной граничной задачей ([2], с. 24) называется задача нахождения решения соотношений

$$
L u=f, \quad \Gamma u \in B,
$$

где $B$ - линейное подпространство в граничном пространстве $C(L)=D(L) / D\left(L_{0}\right)$, определяющее граничную задачу. Задача (7) называется корректной, если оператор $L_{B}=\left.L\right|_{D\left(L_{B}\right)}$, $D\left(L_{B}\right)=\Gamma^{-1} B$, является разрешимым расширением оператора $L_{0}$, т. е. если оператор 
$L_{B}: D\left(L_{B}\right) \rightarrow H^{+}$имеет непрерывный обратный (который является правым обратным к $L$ ). Хорошо известно (М.Й. Вишик [1], в интерпретации Л. Хёрмандера [2], с. 24-25), что у оператора $L_{0}$ существует разрешимое расширение (и для оператора $L$ существует корректная граничная задача (7)) тогда и только тогда, когда выполнены условия (2) и (3). Сопряженной к (7) называется граничная задача $L^{+} v=g, \Gamma^{+} v \in B^{+}$, где пространство $B^{+}=\Gamma^{+} D_{B}^{+}, D_{B}^{+}=\left\{v \in D\left(L^{+}\right) \mid \forall u \in \Gamma^{-1}(B),[u, v]=0\right\}, \quad$ порождено формой Грина $[u, v]=\int_{\Omega}\left(L u \cdot \bar{v}-u \cdot \overline{L^{+} v}\right) d x=<\mathcal{L}_{\partial \Omega} \Gamma u, \Gamma^{+} v>=-<\Gamma u, \mathcal{L}_{\partial \Omega}^{+} \Gamma^{+} v>$.

\section{2. ЗАДАЧА ДИРИХЛЕ}

Определение 1. Обобщенным решением задачи Дирихле для уравнения (1) с правой частью $f \in D^{\prime}\left(L_{0}\right)$ будем называть элемент $u \in D\left(L_{0}\right)$, удовлетворяющий "интегральному" тождеству

$$
<L_{0} u, \mathcal{L} v>_{H^{+}}=<f, v>_{H}
$$

для любого элемента $v \in H_{0}^{l}$ с минимальным оператором $L_{0}$.

Тождество (8) эквивалентно тождеству

$$
\forall v \in D\left(L_{0}\right)<L_{0} u, L_{0} v>_{H^{+}}=<f, v>_{H},
$$

которое можно записать как уравнение

$$
L_{0}^{\prime} L_{0} u=f
$$

где $L_{0}^{\prime}: H^{+} \rightarrow D^{\prime}\left(L_{0}\right)$ - дуальный (в смысле оснащенных пространств) оператор к оператоpy $L_{0}: D\left(L_{0}\right) \rightarrow H^{+}$. Заметим, что в силу плотности вложения $H_{0}^{l} \subset H$ имеется плотность вложения $D\left(L_{0}\right) \subset H$, а потому и плотность вложения $H \subset D^{\prime}\left(L_{0}\right)$, и определение 1 корректно в силу теории оснащенных пространств ([3], с. 45-54), кроме того, можем рассматривать задачу Дирихле (9) с элементом $f \in H$.

Аналогичная постановка задачи Дирихле для уравнения (2) приводит к тождеству

$$
<L_{0}^{+} u, \mathcal{L}^{+} v>_{H}=<f, v>_{H^{+}}
$$

для любого элемента $v \in H_{0}^{+l}$ и элемента $f \in D^{\prime}\left(L_{0}^{+}\right)$, эквивалентному уравнению

$$
\left(L_{0}^{+}\right)^{\prime} L_{0}^{+} u=f .
$$

Определение 2. Обобщенную задачу Дирихле (8) будем называть корректно поставленной или просто корректной, если для каждого элемента $f \in D^{\prime}\left(L_{0}\right)$ существует единственное обобщенное решение $u \in D\left(L_{0}\right)$, непрерывно зависящее от $f$, т. е. если оператор $L_{0}^{\prime} L_{0}$ : $D\left(L_{0}\right) \rightarrow D^{\prime}\left(L_{0}\right)$ имеет непрерывный двусторонний обратный.

Теорема 2. Для того, чтобы обобщенная задача Дирихле (8) была корректна, необходимо и достаточно условие Вишика (4).

Доказательство. Условие (4) эквивалентно непрерывности оператора $M_{1}: \operatorname{Im} L_{0} \rightarrow D\left(L_{0}\right)$ обратного к оператору $L_{0}: D\left(L_{0}\right) \rightarrow \operatorname{Im} L_{0}$. При этом ядро $\operatorname{ker} L_{0}$ тривиально, а образ $\operatorname{Im} L_{0}$ замкнут в $H^{+}$, отсюда следует, что $H^{+}$- дуальное пространство к $\operatorname{Im} L_{0}$ - можно отождествить с ним самим, и оператор $M_{1}^{\prime}: D^{\prime}\left(L_{0}\right) \rightarrow \operatorname{Im} L_{0}$ является непрерывным обратным к оператору $L_{0}^{\prime}: \operatorname{Im} L_{0} \rightarrow D^{\prime}\left(L_{0}\right)$. Таким образом из условия (4) получаем существование непрерывного оператора $M=M_{1} M_{1}^{\prime}$, обратного к оператору $L_{0}^{\prime} L_{0}: D\left(L_{0}\right) \rightarrow D^{\prime}\left(L_{0}\right)$. Наоборот, существование непрерывного оператора $M: D^{\prime}\left(L_{0}\right) \rightarrow D\left(L_{0}\right)$, обратного к оператору $L_{0}^{\prime} L_{0}$, влечет равенство $M L_{0}^{\prime} L_{0}=\left.\mathrm{id}\right|_{D\left(L_{0}\right)}$, откуда видно, что оператор $M L_{0}^{\prime}$ является непрерывным левым обратным к $L_{0}$. 
Заметим только, что ядром оператора $M L_{0}^{\prime}: H^{+} \rightarrow D\left(L_{0}\right)$ является пространство $H^{+} \ominus$ $\operatorname{Im} L_{0}=\operatorname{ker} L^{+}$, где $\ominus$ обозначает ортогональную разность.

Аналогично доказывается

Теорема 3. Для того, чтобы обобщенная задача Дирихле (10) была корректна, необходимо и достаточно условие (5).

Пример 2. Рассмотрим обобщенную задачу Дирихле для уравнения Пуассона $-\Delta u=f$ в произвольной области $\Omega \subset \mathbb{R}^{n}$. Здесь $\mathcal{L}=\operatorname{grad}, \mathcal{L}_{\circ}^{+}=-\operatorname{div}, H=L_{2}(\Omega), H^{+}=L_{2}^{n}(\Omega)$, $D(L)=W_{2}^{1}(\Omega), D\left(L_{0}\right)=W_{2}^{1}(\Omega), f \in D^{\prime}\left(L_{0}\right)=\left[W_{2}^{1}(\Omega)\right]^{\prime}$ в стандартных обозначениях. Тогда определение 1 сводится к общепринятому определению обобщенного решения задачи Дирихле для уравнения Пуассона [10]. Теорема 1 утверждает, что корректность обобщенной задачи Дирихле для этого уравнения в области $\Omega$ имеет место тогда и только тогда, когда в этой области выполнено неравенство Фридрихса

$$
\exists C>0, \forall \varphi \in C_{0}^{\infty}(\Omega) \quad\|\nabla \varphi\|_{L_{2}^{n}(\Omega)}>C\|\varphi\|_{L_{2}(\Omega)},
$$

которое представляет собой формулировку условия Вишика (4) для оператора $\mathcal{L}=\operatorname{grad}$. Известно [10], что неравенство Фридрихса выполнено в любой области, лежащей в некоторой полосе, например, в полосе $\Pi=\left\{x \in \mathbb{R}^{n}, a<x_{n}<b\right\}$. Ненамного сложнее случай равно-

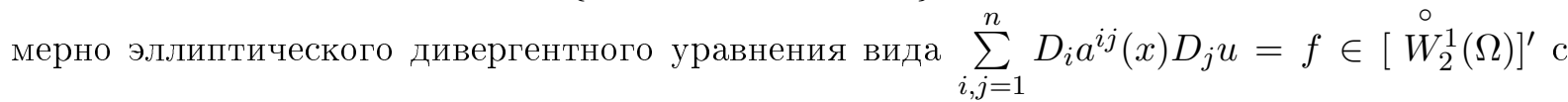
симметрической вещественной положительно определенной матрицей $\left(a^{i j}\right), a^{i j} \in C^{1}(\bar{\Omega})$, $\sum_{i j} a^{i j}(x) \xi_{i} \xi_{j} \geq \alpha|\xi|^{2}, \alpha=$ const $>0$. Здесь $\mathcal{L} u=\sum_{j} b^{i j}(x) D_{j} u, B=\left(b^{i j}\right)=\sqrt{A}>0$, $\mathcal{L}^{+} \cdot=\sum_{j} D_{i}\left(b^{i j}(x) \cdot\right)$, а пространства и выводы те же, что и в случае уравнения Пуассона.

Из теоремы 1 следует

Теорема 4. Если $\mathcal{L}$ - один из операторов классов i)-v) в ограниченной области, то обобщенная задача Дирихле (8) для уравнения (1) корректна.

Пример 3. Рассмотрим еще один пример векторного дифференциального оператора $\mathcal{L}=$ $\left(\mathcal{L}_{1}, \ldots, \mathcal{L}_{N}\right)$ в пространствах $H=L_{2}(\Omega), H^{+}=L_{2}^{N}(\Omega)$, где один из скалярных операторов $\mathcal{L}_{j}$, например $\mathcal{L}_{1}$, удовлетворяет в заданной области $\Omega$ условию $(4)$ в $L_{2}(\Omega)$. Тогда и сам оператор $\mathcal{L}$ удовлетворяет в этой области условию (4). Действительно, для нормы графика

$$
\|\varphi\|_{L}^{2}=\|\varphi\|_{L_{2}(\Omega)}^{2}+\sum_{j}\left\|\mathcal{L}_{j} \varphi\right\|_{L_{2}^{N}(\Omega)}^{2} \geq C\|\varphi\|_{L_{2}(\Omega)}^{2}, \quad C>1
$$

если

$$
\left\|\mathcal{L}_{1} \varphi\right\|_{L_{2}^{N}(\Omega)} \geq C_{1}\|\varphi\|_{L(\Omega)}, \quad C_{1}>0 .
$$

Таким образом, условие (4) выполнено для оператора $\mathcal{L}$ и по теореме 4 обобщенная задача Дирихле для уравнения $\sum_{j=1}^{N} \mathcal{L}_{j}^{+} \mathcal{L}_{j} u=f$ корректна в области $\Omega$.

Замечание 1. Обобщенная задача Дирихле $(8)$ помогает найти вид проектора $\Gamma_{\operatorname{Im}}$ и проектора $P_{0}=i_{0}^{-1}: D(L) \rightarrow D\left(L_{0}\right)$ из диаграммы (6). Именно, при условии (4) определим $v=P_{0} u$ для данного $u \in D(L)$ как решение обобщенной задачи Дирихле для уравнения (1.1) вида $L_{0}^{\prime} L_{0} v=L_{0}^{\prime} L u$. Ядро оператора $L_{0}^{\prime}$ совпадает с ядром $\operatorname{ker} L^{+}$, поэтому $L_{0}^{\prime} L u=0$ при $u \in \operatorname{ker} L+W$, где $L W=\operatorname{ker} L^{+}$, а на $D\left(L_{0}\right) P_{0}^{2}=P_{0}$. Кроме того, при том же условии 
(4) сопоставление $U \in H^{+} \rightarrow P_{\operatorname{Im}} U$, где $P_{\operatorname{Im}} U=L_{0} u$, а $u$ - решение обобщенной задачи Дирихле для уравнения (1) вида $L_{0}^{\prime} L_{0} u=L_{0}^{\prime} U$, является ортогональным проектором на $\operatorname{Im} L_{0}$ и $\Gamma_{\mathrm{Im}}=1-P_{\mathrm{Im}}$. Это наблюдение позволяет привести достаточное условие для выполнения свойства

$$
\text { гладкое ядро } \tilde{K}^{+}=\operatorname{ker} L^{+} \cap H^{l} \text { плотно в ядре } \operatorname{ker} L^{+} \subset H^{+} \text {. }
$$

Если существует множество $\mathcal{E}$, плотное в $H^{+l}$ и такое, что $P_{\operatorname{Im}} \mathcal{E} \subset H^{+l}$, то условие $(11)$ выполнено. Действительно, тогда множество $\mathcal{E}$ плотно в $H^{+}$, а $\left(1-P_{\mathrm{Im}}\right) \mathcal{E} \subset H^{+l}$ плотно в $\operatorname{ker} L^{+}$.

Замечание 2. Пусть подпространство $A$ пространства $D(L)$ ортогонально пространству $D\left(L_{0}\right)$ (аналогично подпространство $A^{+}$пространства $D\left(L^{+}\right)$ортогонально пространству $\left.D\left(L_{0}^{+}\right)\right)$. Тогда ясно, что граничную задачу $(7)$ можно определять как подпространство пространства $A$. Кроме того, как показано в работе [11], $L: A \rightarrow A^{+}, L^{+}: A^{+} \rightarrow A$, и $\forall u \in A L^{+} L u=-u, \forall v \in A^{+} L L^{+} v=-v$, т. е. $A=\operatorname{ker}\left(L^{+} L+I\right), A^{+}=\operatorname{ker}\left(L L^{+}+I\right)$. Обобщенная задача Дирихле (8) помогает найти вид проектора $P_{A}: D(L) \rightarrow D\left(L_{0}\right)$ с ядром $A$. Рассмотрим на гладком пространстве $H^{l}$ операцию вида $\mathcal{M} u=\left(\begin{array}{c}\mathcal{L} u \\ u\end{array}\right)$, тогда $\mathcal{M}^{+}\left(\begin{array}{c}v_{1} \\ v_{2}\end{array}\right)=$ $\mathcal{L}^{+} v_{1}+v_{2}, D(M)=D(L), D\left(M_{0}\right)=D\left(L_{0}\right)$. Для этой операции выполнено условие $(4)$, как в примере 3 , и по теореме 2 корректна задача Дирихле (8) с оператором $M$. Оператор $1-P_{A}$, действуюший по правилу $D(L) \ni u \rightarrow v$, где $v$ - решение задачи Дирихле для уравнения (1) вида $M_{0}^{\prime} M_{0} v=\left(\mathcal{L}^{+} L+1\right) u\left(=\mathcal{M}^{\prime} M u\right)$, является ортогональным проектором с ядром $A$ из пространства $D(L)$ на $D\left(L_{0}\right)$.

Замечание 3. Пусть оператор $\tilde{L}$ определен как замыкание оператора $\mathcal{L}$, первоначально заданного на $\left(C^{\infty}(\bar{\Omega})\right)^{j}$, в норме графика $\|u\|_{L}^{2}=\|u\|_{L_{2}^{j}(\Omega)}^{2}+\|\mathcal{L} u\|_{L_{2}^{k}(\Omega)}^{2}$, и аналогично определен оператор $\tilde{L}^{+}$. Условия

$$
\begin{aligned}
& \tilde{L}=\left(L_{0}^{+}\right)^{*}, \\
& \tilde{L}^{+}=\left(L_{0}\right)^{*}
\end{aligned}
$$

играют важную роль в общей теории граничных задач ([5], с. 15 и ниже; [3], с. 93-98). Обобщенная задача Дирихле (8) помогает найти достаточные условия для выполнения условий (12) и (13). Рассматривая задачу Дирихле для уравнения $\mathcal{R}^{+} \mathcal{R} v=\mathcal{R}^{+} \mathcal{R} u$ с операцией $\mathcal{R}=\mathcal{L} L^{+}+1$ можем так же, как и в замечании 1 , ввести условие

$$
\text { существует множество } \mathcal{E}, \text { плотное в } H^{+2 l} \text { и такое, что } P_{R \operatorname{Im}} \mathcal{E} \subset H^{+2 l}
$$

с исходным гладким пространством $H^{+2 l} \subset H^{+l}$ для операции $\mathcal{R}$. Такое условие дает плотность гладкого пространства в ядре $\operatorname{ker} R$ с топологией из $H^{+}$. Это вместе с условием

$$
\forall u \in A \quad\|L u\|_{H^{+}} \geq c_{1}\|u\|_{H}
$$

влечет следующие факты:

1) плотность гладкого соболевского пространства $H^{+2 l}$ в пространстве $A^{+}$в топологии $\left.D\left(L^{+}\right), 2\right)$ условие $(13)$, поскольку в $D\left(L_{0}\right)$ плотны гладкие финитные элементы, 3) условие $(12)$, если считать, что $\mathcal{L}^{+}\left(H^{+2 l}\right) \subset H^{+l}$, так как $L^{+} A^{+}=A$. Для скалярного дифференциального оператора с постоянными коэффициентами $\mathcal{L}$ записанное выше условие выполнено в любой ограниченной области, если принять $\mathcal{E}=C^{\infty}(\bar{\Omega})$. Действительно, пусть $m \in \mathbb{Z}_{+}-$любое натуральное число или нуль. Выберем пространства из начала статьи так: $H=H^{+}=H^{m}(\Omega) ; H^{2 l}=H^{+2 l}=H^{m+2 l}(\Omega) ; H_{0}^{2 l}=H_{0}^{+2 l}=H^{m+2 l}(\Omega) \cap H_{0}^{2 l}(\Omega)$; операцию $\mathcal{R}$ порядка $2 l$, заданную как выше с помощью операции $\mathcal{L}$ порядка $l$. Теперь можем строить теорию расширений и граничных задач, как в работах ([1]; [2], с.13-29; [3], с.93-114), а 
также теорию обобщенно поставленной задачи Дирихле настоящего пункта, которая корректна, если выполнено условие Вишика (4). Условие (4) равносильно оценке Хёрмандера

$$
\forall u \in C_{0}^{\infty}(\Omega) \quad\|L u\|_{H^{+}} \geq c_{1}\|u\|_{H}
$$

которая в нашем случае следует из неравенства $\left\|\operatorname{grad}^{m} \mathcal{R} \phi\right\|_{L_{2}(\Omega)} \geq C\left\|\operatorname{grad}^{m} \phi\right\|_{L_{2}(\Omega)} \quad \forall \phi \in$ $C_{0}^{\infty}(\Omega)$. Последнее неравенство в силу перестановочности $\operatorname{grad}$ и $\mathcal{R}$ вытекает из оценки Хёрмандера для оператора $\mathcal{R} D^{\alpha},|\alpha|=m$, в ограниченной области. Отсюда, в частности, следует

для оператора с постоянными коэффициентами в любой ограниченной области условие (12) о плотности гладких функций в $D(L)$ будет выполнено, как только будет выполнено условие (14) о совпадении топологий пространств $D(L)$ и $H^{+}$на пространстве $A$.

\section{3. ЗАДАЧА НЕЙМАНА}

Определение 3. Обобщенным решением задачи Неймана для уравнения (1) с правой частью $f \in D^{\prime}(L)$ будем называть элемент $u \in D(L)$, удовлетворяющий "интегральному" тождеству

$$
<L u, L v>_{H^{+}}=<f, v>_{H}
$$

для любого элемента $v \in D(L)$ с максимальным оператором $L$.

Если оператор $L$ удовлетворяет условию (12), то в последнем определении достаточно требовать удовлетворения тождества (15) для любого элемента $v \in H^{l}$. Тождество (1.7) эквивалентно уравнению

$$
L^{\prime} L u=f
$$

где $L^{\prime}: H^{+} \rightarrow D^{\prime}(L)$ - дуальный (в смысле оснащенных пространств) оператор к оператору $L: D(L) \rightarrow H^{+}$.

В силу плотности вложения $H^{l} \subset H$ имеется плотность вложения $D(L) \subset H$, а потому и плотность вложения $H \subset D^{\prime}(L)$, и определение 2 корректно в силу теории оснащенных пространств ([3], с. 27), кроме того, можем рассматривать задачу Неймана (15) с элементом $f \in H$.

Определение 4. Обобщенную задачу Неймана (15) будем называть нормалъно корректной, если для каждого элемента $f \in D^{\prime}\left(L_{0}\right), H$-ортогонального к пространству $\operatorname{ker} L \subset D(L)$, существует единственный с точностью до аддитивной добавки $h \in \operatorname{ker} L$ элемент $u \in D(L)$, являющийся решением уравнения (16), который непрерывно зависит от $f$. Другими словами, задача (15) нормально корректна, если существует непрерывный оператор $N$, обратный к оператору $\hat{L}: D(L) / \operatorname{ker} L \rightarrow D_{\perp \operatorname{ker} L}^{\prime}(L)=\left\{f \in D^{\prime}(L) \mid f \perp \operatorname{ker} L\right\}$, индуцированному оператором $L^{\prime} L: D(L) \rightarrow D^{\prime}(L)$ на фактор-пространстве по ядру.

Теорема 5. Обобщенная задача Неймана (15) нормалъно корректна тогда и только тогда, когда оператор L нормально разрешим:

$$
\text { образ } \operatorname{Im} L \text { оператора } L: D(L) \rightarrow H^{+} \text {замкнут в } H^{+} \text {. }
$$

В частности, это так, если выполнено условие (5).

Доказательство аналогично доказательству теоремы 1 с той разницей, что вначале следует провести факторизацию $D(L) / \operatorname{ker} L, D^{\prime}(L) / \operatorname{coker} L^{\prime}$, а затем использовать те же рассуждения.

Определение 5. Будем называть обобщенную задачу Неймана (15) фредголъмовой, если оператор $L^{\prime} L$ фредгольмов, т. е. если он непрерывен, нормально разрешим и, кроме того, размерности ядра и коядра конечны и равны между собой. 
Теорема 6. Для того чтобы обобщенная задача Неймана (15) была фредголвмовой, необходимо и достаточно, чтобы было выполнено условие нормальной разрешимости оператора $L$ (17) и чтобы ядро $\operatorname{ker} L$ было конечномерным.

Доказательство. Достаточность. Заметим сначала, что образ оператора $L^{\prime}$ в пространстве в силу нормальной разрешимости оператора $L D^{\prime}(L)$ состоит из элементов, ортогональных ядру $\operatorname{ker} L \subset D(L)$, а коядро $\operatorname{coker} L=\left(\operatorname{ker} L_{0}\right)^{\prime}$ изоморфно $\operatorname{ker} L_{0}$ в силу конечномерности, что доказывает достаточность. Необходимость. Фредгольмовость оператора $L^{\prime} L_{0}$ влечет, что оператор $\hat{L}: D(L) / \operatorname{ker} L \rightarrow D_{\perp \operatorname{ker} L}^{\prime}(L)$, порожденный оператором $L^{\prime} L$ и действующий по формуле $\hat{L}(u+\operatorname{ker} L)=L^{\prime} L u$, является изоморфизмом, и тогда, рассуждая как в доказательстве теоремы 2 , получим замкнутость образа $\operatorname{Im} L$, откуда следует нормальная разрешимость оператора $L^{\prime}: \operatorname{Im} L \rightarrow D^{\prime}(L)$ и изоморфность коядра оператора $L^{\prime}$ ядру оператора $L$.

Из теоремы 2 и 5 вытекает

Теорема 7. Если обобщенная задача Дирихле (10) для уравнения (2) корректна, то обобщенная задача Неймана (15) для уравнения (1) нормально корректна.

Пример 4. Рассмотрим обобщенную задачу Неймана для уравнения Пуассона $-\Delta u=f$. Операторы $\mathcal{L}, \mathcal{L}^{+}, L$ и пространства $H, H^{+}, D(L)$ определим как в примере 1 . Теорему 7 не можем использовать, поскольку задача Дирихле для уравнения $-\operatorname{grad} \operatorname{div} \bar{u}=f$ не корректна $(\bar{u}=\operatorname{rot} \bar{v}$ с любым гладким $\bar{v}$ лежит в ядре этого оператора). Теорему 5 можно использовать, если ввести дифференциальные когомологии по де Раму внешних форм с коэффициентами из $L_{2}(\Omega)$, как это сделано в $([15]$, с. 42$) d_{1} f(=\operatorname{grad} f)=\partial_{i} f d x_{i}, d_{2} \omega(=$ $\operatorname{rot} \omega)=\left(\partial_{i} \omega_{j}-\partial_{j} \omega_{i}\right) d x_{i} \wedge d x_{j}$. Также заметим, что непрерывный оператор $d_{2}: L_{2}^{n}(\Omega) \rightarrow$ $\left(W_{2}^{1, n}(\Omega)^{\prime}\right) \wedge\left(W_{2}^{1, n}(\Omega)^{\prime}\right)$ имеет замкнутое ядро ker $d_{2} \subset L_{2}^{n}(\Omega)$, которое по теореме де Рама отличается от образа $d_{1}$ на пространство первых когомологий $H^{1}(\Omega)$. Поэтому если область $\Omega$ такова, что $H^{1}(\Omega, \mathbb{R})$ конечномерно, то и образ оператора $\mathcal{L}=\operatorname{grad}$ замкнут, и задача Неймана (15) для уравнения Пуассона нормально корректна. Здесь $\operatorname{ker} L=\{$ const $\}, D_{\perp}^{\prime}(L)=$ $\left\{u \in\left(W_{2}^{1}(\Omega)\right)^{\prime} \mid \int_{\Omega} u d x=0\right\}$.

$\mathrm{C}$ другой стороны, нетрудно показать, что нормальная разрешимость оператора $L$ эквивалентна выполнению неравенства

$$
\exists C>0 \quad \forall u \in D(L) \quad\|u\|_{L_{2}(\Omega)}^{2}-\left\|P_{\text {ker }} u\right\|_{L_{2}(\Omega)}^{2} \leq C\|L u\|_{L_{2}(\Omega)}^{2},
$$

где $P_{\text {ker }}: L_{2}(\Omega) \rightarrow \operatorname{ker} M-$ ортопроектор. Для $L=\nabla$ имеем $\operatorname{ker} L=\{$ const $\}, P_{\text {ker }}: u \rightarrow$ $\frac{1}{\operatorname{meas} \Omega} \int_{\Omega} u(x) d x$ и последнее неравенство в этом случае имеет вид известного неравенства Пуанкаре

$$
\exists C>0 \quad \forall u \in C^{\infty}(\bar{\Omega}) \quad\|u\|_{L_{2}(\Omega)}^{2} \leq \frac{1}{\operatorname{meas} \Omega}\left(\int_{\Omega} u d x\right)^{2}+C\|\nabla u\|_{L_{2}(\Omega)}^{2} .
$$

Таким образом, по теореме 5 обобщенная задача Неймана для уравнения Пуассона нормально корректна в области тогда и только тогда, когда в этой области выполнено неравенство Пуанкаре, справедливое в области $\Omega$, у которой $\operatorname{dim} H^{1}(\Omega, \mathbb{R})<\infty$.

Пример 5. Рассмотрим классы дифференциальных операторов, перечисленные в примере 1. Из теорем 4 и 5 вытекает

Теорема 8. Если $\mathcal{L}$ - один из операторов классов i)-v) из примера 1, то обобщеннал задача Неймана (15) нормально корректна. 


\section{4. ДРУГИЕ ЛИНЕЙНЫЕ ГРАНИЧНЫЕ ЗАДАЧИ}

Пусть линейное подпространство $B \subset C(L)$ задает граничное условие $\Gamma u \in B$, как во введении, и расширение $L_{B}$ оператора $L_{0}$. Пусть $B^{+} \subset C\left(L^{+}\right)$- линейное подпространство, задающее граничную задачу $L^{+} v=g, \Gamma v \in B^{+}$, сопряженную к задаче (7).

Определение 6. Обобщенным решением граничной задачи $\Gamma u \in B, \Gamma^{+} L u \in B^{+}$, порожденной граничной задачей (7), для уравнения (1) с правой частью $f \in D^{\prime}\left(L_{B}\right)$ будем называть элемент $u \in D\left(L_{B}\right)$, удовлетворяющий "интегральному" тождеству

$$
<L_{B} u, L_{B} v>_{H^{+}}=<f, v>_{H}
$$

для любого элемента $v \in D\left(L_{B}\right)$.

Тождество (18) можно записать в виде уравнения

$$
L_{B}^{\prime} L_{B} u=f .
$$

Определение 7. Обобщенную граничную задачу (18) будем называть нормально корректной, если существует непрерывный оператор $R$, обратный к оператору

$$
\widehat{L_{B}^{\prime} L_{B}}: D\left(L_{B}\right) / \operatorname{ker} L_{B} \rightarrow D_{\perp \operatorname{ker} L_{B}}^{\prime}\left(L_{B}\right)=\left\{f \in D^{\prime}\left(L_{B}\right) \mid f \perp \operatorname{ker} L_{B}\right\} .
$$

Граничную задачу (18) будем называть корректной, если оператор $L_{B}^{\prime} L_{B}: D\left(L_{B}\right) \rightarrow D^{\prime}\left(L_{B}\right)$ - изоморфизм, т. е. если эта задача нормально корректна, а ядро и коядро оператора $L_{B}^{\prime} L_{B}$ тривиальны, и будем называть эту задачу фредгольмовой, если она нормально корректна, и, кроме того, ядро и коядро оператора $L_{B}^{\prime} L_{B}$ конечномерны.

Теорема 9. Обобщеннал граничнал задача (18) нормалъно корректна тогда и толъко тогда, когда оператор $L_{B}$ нормально разрешим; фредгольмова тогда и толъко тогда, когда она нормалъно корректна и ядро $\operatorname{ker} L_{B}$ конечномерно; корректна тогда и толъко тогда, когда она нормально корректна $u \operatorname{ker} L_{B}=0$.

Доказательство теоремы аналогично доказательству теорем 4 и 5.

Пример 6. Рассмотрим обобщенно поставленную задачу (18) с волновым оператором $L=$ $\square=\frac{\partial^{2}}{\partial x \partial y}=L^{+}$в единичном круге $\Omega$ и с пространством $B$, порожденным граничным условием

$$
\left.u\right|_{\Gamma_{1}}=0,\left.u_{\nu}^{\prime}\right|_{\Gamma_{2}}=0
$$

где $\Gamma_{1}=\{\pi / 2 \leq \tau \leq 2 \pi\}, \Gamma_{2}=\{\pi \leq \tau \leq 3 \pi / 2\}, \tau-$ угловая переменная. Определим пространство $D\left(\square_{B}\right)$ как замыкание множества гладких в $\bar{\Omega}$ функций, удовлетворяющих условию (19), в норме графика $\|\cdot\|_{\square}$. Для гладкой функции $u$ в виду того, что $L$-следы на окружности обращаются в нуль (см. определения и выражения для $L$-следов в работе [11]) тогда и только тогда, когда обычные следы на ней обращаются в нуль. Получаем, что задачу (17) можно записать в виде

$$
\square^{2} u=f,\left.\quad u\right|_{\Gamma_{1}}=0,\left.\quad u_{\nu}^{\prime}\right|_{\Gamma_{2}}=0,\left.\quad \square u\right|_{C \Gamma_{1}}=0,\left.\quad(\square u)_{\nu}^{\prime}\right|_{C \Gamma_{2}}=0,
$$

где $C \Gamma_{j}=\partial K \backslash \Gamma_{j}-$ дополнения множеств $\Gamma_{j}$ и вторая пара условий задает условия $\Gamma^{+} \mathcal{L} u \in$ $B^{+}$. В работе [11] доказано, что задача (19) для уравнения $\frac{\partial^{2} u}{\partial x \partial y}=f$ корректна, откуда следует $\operatorname{Im} \square_{B}=L_{2}(K)$ и $\operatorname{ker} \square_{B}=0$, поэтому теорема 9 дает корректность такой обобщенной задачи.

Рассмотрим множество нормально корректных задач. В частности, задачу Дирихле (8) будем называть нормально корректной, если образ $\operatorname{Im} L_{0}$ замкнут в пространстве $H^{+}$. Это 
означает, что оператор $\widehat{L_{0}^{\prime} L_{0}}: D\left(L_{0}\right) / \operatorname{ker} L_{0} \rightarrow D_{\perp \text { ker } L_{0}}^{\prime}\left(L_{0}\right)=\left\{f \in D^{\prime}\left(L_{0}\right) \mid f \perp\right.$ ker $\left.L_{0}\right\}$ имеет непрерывный обратный.

Предположим, что выполнено условие (17) нормальной разрешимости оператора $L_{0}$ :

$$
\text { пространство } \operatorname{Im} L_{0}-\text { замкнуто в } H^{+}
$$

Тогда любое нормально разрешимое расширение $L_{B}$ порождает диаграмму

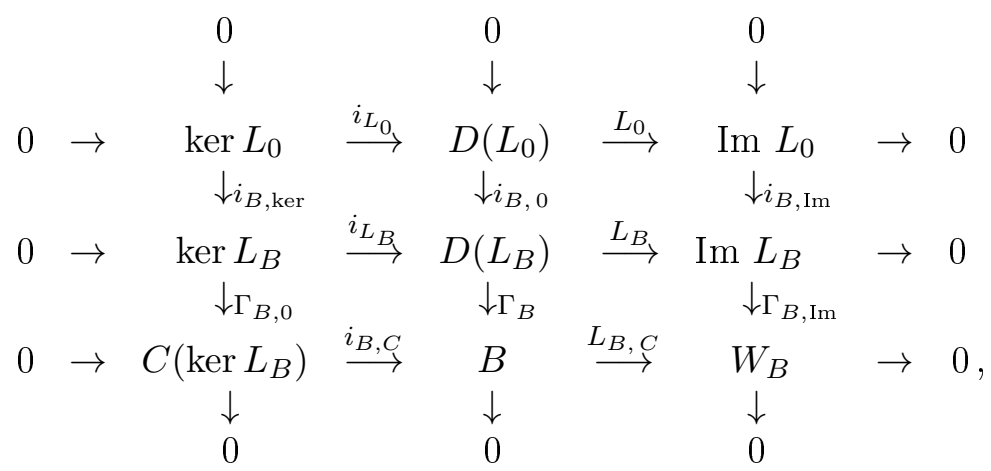

которая означает разложение в прямую сумму $L_{B}=L_{0} \oplus L_{B, C}$. Условие замкнутости $\operatorname{Im} L_{0}$ в $\operatorname{Im} L_{B}$ в силу гильбертовости пространств необходимо и достаточно для полноты всех пространств и непрерывности операторов диаграммы (20). Кроме того, из корректности граничной задачи (7) имеем разложение в прямую сумму $B=C\left(\operatorname{ker} L_{B}\right) \oplus B_{1}$ с некоторым $B_{1}$.

Рассматривая диаграмму дуальных объектов и операторов, означающую разложение $L_{B}^{\prime}=L_{0}^{\prime} \oplus L_{B, C}^{\prime}$ (дуальность по отношению к центральным пространствам $H$ и $H^{+}$) и сравнивая эти две диаграммы в силу замкнутости в $H^{+}$пространств стоящих в последнем столбце диаграммы (20), получим разложение в прямую сумму

$$
L_{B}^{\prime} L_{B}=L_{0}^{\prime} L_{0} \oplus L_{B, C}^{\prime} L_{B, C} .
$$

Если теперь $\operatorname{ker} L_{B}=0$, т. е. если задача (18) корректна, то, как видно из диаграммы (20), ker $L_{0}=0$, что вместе с условием $\left(17_{0}\right)$ дает выполнение условия (4) и корректность задачи Дирихле. В этом случае левый столбец диаграммы (20) тривиален и оператор $L_{B, C}-$ изоморфизм. Но тогда и оператор $L_{B, C}^{\prime} L_{B, C}: B \rightarrow B^{\prime}-$ изоморфизм. Здесь пространство $B^{\prime}-$ $H$-дуальное пространство к пространству $B$, вложенному в $D(L)$ с помощью некоторого оператора $\hat{\Gamma}_{B}$, правого обратного к оператору факторизации $\Gamma_{B}$.

Доказана

Теорема 10. Пусть выполнено предположение $\left(17_{0}\right)$, т.е. нормально корректна задача Дирихле (8).

1) Если задача (18) нормалъно корректна, то справедливо разложение в прлмуюо сумму (21) с оператором $L_{B, C}^{\prime} L_{B, C}: B \rightarrow B^{\prime}$.

2) Если задача (18) корректна, то корректна задача Дирихле и в разложении (21) опеpaтор $L_{B, C}^{\prime} L_{B, C}: B \rightarrow B^{\prime}-$ изоморфизм.

Справедливо и обратное утверждение, описывающее все корректные обобщенные граничные задачи (18).

Теорема 11. Пусть выполнены предположсения $\left(5^{\prime}\right),\left(17_{0}\right)$. Каждое подпространство В $\subset$ $C(L)$ со свойством (21) порождает корректную граничную задачу (18). 
Доказательство. Условия $\left(5^{\prime}\right),\left(17_{0}\right)$ позволяют построить диаграмму (6) в категории гильбертовых пространств. Подпространство $B$ со свойством (21) порождает разложение в прямую сумму $C\left(L_{B}\right):=\overline{C(\operatorname{ker} L)+B}=C(\operatorname{ker} L) \oplus B$ и замкнутое расширение $L_{B}: D\left(L_{B}\right)=$ $\Gamma^{-1} B$ оператора $L_{0}$. Это расширение нормально разрешимо, поскольку оператор

$$
\hat{L}: D(L) / \operatorname{ker} L \rightarrow H^{+},
$$

порожденный оператором $L$ факторизацией по ядру, является изоморфизмом по условию $\left(5^{\prime}\right)$, и поэтому по утвеждению 9 соответствующая задача (18) корректна.

Диаграмма (21), построенная в предположениях $\left(5^{\prime}\right),\left(17_{0}\right)$, означает разложение в прямую сумму

$$
D\left(L_{B}\right)=\operatorname{ker} L_{0} \oplus W_{B} \oplus C\left(\operatorname{ker} L_{B}\right) \oplus \operatorname{Im} L_{0},
$$

поэтому получим, что нормально разрешимое расширение $L_{B}$ оператора $L_{0}$ параметризуется парой операторов

$$
V_{0}: \operatorname{Im} L_{0} \rightarrow \operatorname{ker} L_{0}, V_{C}: \Gamma_{\operatorname{Im}} \operatorname{Im} L_{B}=\left(\operatorname{ker} L^{+} \cap \operatorname{Im} L_{B}\right) \rightarrow C\left(\operatorname{ker} L_{B}\right) .
$$

Получена

Теорема 12. В предположениях $\left(5^{\prime}\right),\left(17_{0}\right)$ множество всех нормально разрешимых расширений оператора $L_{0}$ и, значит, множество всех нормально разрешимых обобщенно поставленных граничных задач (18) параметризуется тройкой $\left(T, V_{0}, V_{C}\right)$, где $T \subset H^{+}(=$ $\left.\operatorname{Im} L_{\mathrm{B}}\right)$ - замкнутое подпространство, включающее $\operatorname{Im} L_{0}, V_{0}: \operatorname{Im} L_{0} \rightarrow \operatorname{ker} L_{0} u V_{C}:$ $\Gamma_{\operatorname{Im}} T \rightarrow \operatorname{ker} L \ominus \operatorname{ker} L_{0}-$ пара непрерывных операторов.

\section{ЛитератУра}

[1] Вишик М.Й. Об общих краевых задачах для эллиптических дифберенциалънъх уравнений, Тр. Моск. Матем. о-ва, №1, 187-246 (1952).

[2] Хёрмандер Л. К теории общих дифберенциалънъх операторов в частных производных (ИЛ, М., 1959).

[3] Березанский Ю.М. Разложение по собственным функииям самосопряљенных операторов (Наук. думка, Киев, 1965).

[4] Агранович М.С. Об уравнениях в частных производных с постоянными коэфбициентами, Успехи матем. наук 16(2), 27-93 (1961).

[5] Дезин А.А. Общие вопросы теории граничнъх задач (Наука, М., 1980).

[6] Петровский И.Г. О некоторых проблемах теории уравнений с частными производными. Успехи матем. наук 1 (3-4), 44-70 (1946).

[7] Лопатинский Я.Б. Об одном способе сведения краевых задач для систем дифференциалъных уравнений эллиптического типа к регулярным интегралъным уравнениям, Укр. матем. журн. 5 (2), 123-151 (1953).

[8] Бицадзе А.В. Некоторые классы дифференциалъных уравнений с частными производными (Наука, М., 1981).

[9] Солдатов А.П. Сингулярные интегралъные операторы и эллиптические краевые задачи. I, Функциональный ан., СМФН 63 (1), 1-189 (2017).

[10] Ладыженская О.А. Краевые задачи математической физики (ФМ, М., 1973).

[11] Бурский В.П. О граничнъх свойствах решений дифференциалъных уравнений и общих граничнъх задачах, Тр. Московск. Матем. о-ва, № 68, 185-225 (2007).

[12] Бурский В.П. Обобщенные решения граничных задач для дифберенщиалъных операторов общего вида, УMH 53(4), 215-216 (1998).

[13] Burskii V. On well-posedness of boundary value problems for some class of general PDEs in a generalized setting, Functional Diff. Equ. 8 (1-2), 89-100 (2001).

[14] Маклейн С. Гомология (Мир, М., 1966).

[15] Уитни Х. Геометрическая теория интегрирования (ИЛ, М., 1960). 
Владимир Петрович Бурский

Московский физико-технический институт,

Институтский пер., д. 9, г. Долгопрудный, 141701, Россия,

e-mail : bvp30@mail.ru

\section{V.P. Burskii}

\section{Generalized solutions of the linear boundary value problems}

Abstract. We use constructions from the general theory of boundary value problems to build a theory of generalized boundary value problems for the generalized Poisson equation. Namely, generalized solutions of various boundary value problems are introduced and studied for the matrix generalization of the Poisson equation, a description of the set of all such well-posed problems is given. The results obtained are used for advancements in the original general theory of boundary value problems.

Keywords: general theory of boundary value problems, extensions of differential operators, generalized statements, generalized solutions.

Vladimir Petrovich Burskii

Moscow Institute of Physics and Technology, 9 Institutskiy per., Dolgoprudny, 141701 Russia,

e-mail: bvp30@mail.ru 